\title{
Ocorrência de parasitos gastrintestinais em cães (Canis familiaris) com diarréia aguda oriundos da região metropolitana de Londrina, Estado do Paraná, Brasil
}

\author{
Occurrence of gastrointestinal parasites in dogs (Canis familiaris) \\ with acute diarrhea from metropolitan region of \\ Londrina, Parana State, Brazil
}

\author{
Fábio Augusto Galli dos Santos ${ }^{1}$; Milton Hissashi Yamamura²; \\ Odilon Vidotto ${ }^{2}$; Pedro Luiz de Camargo ${ }^{3 *}$
}

Resumo

\begin{abstract}
Foram examinadas 150 amostras de fezes colhidas de cães com diarréia aguda (Grupo 1), e outras 50 de animais sem diarréia (Grupo 2), atendidos entre outubro de 2004 e outubro 2005, no Hospital Veterinário Escola da Universidade Estadual de Londrina. Dos 150 animais do G1, 44 (29,3\%) estavam infectados por helmintos e/ou protozoários, sendo que destes, 32 (72,7\%) apresentavam infecção simples e 12 (27,3\%) infecções múltiplas. Os gêneros identificados nestes animais foram: Giardia sp. (9,3\%), Cystoisospora sp. (7,3\%), Ancylostoma sp. (7,3\%), Entamoeba sp. (6,7\%), Toxocara sp. (4\%), Pentatrichomonas hominis $(3,3 \%)$ e Trichuris vulpis (1,3\%). Dos 50 animais sem diarréia, seis (12\%) eram positivos, dois (4\%) para Ancylostoma sp., três (6\%) para Giardia sp. e um (2\%) para Ancylostoma sp. e Cystoisospora simultaneamente. Houve relação estatisticamente significativa $(\mathrm{P}=0,005)$ entre o parasitismo e a ocorrência de diarréia, assim como entre a faixa etária e a proporção de infecções $(\mathrm{P}<0,001)$, porém, machos e fêmeas foram igualmente acometidos $(\mathrm{P}=0,08)$. O número de cães com raça definida foi significativamente maior do que de cães sem definição racial $(\mathrm{P}=0,01)$. Os resultados demonstram a importância de realização periódica de exames parasitológicos em cães com e sem diarréia para tratamento específico e adoção medidas de controle e profilaxia.
\end{abstract}

Palavras-chave: Cães, diarréia aguda, parasitos gastrintestinais, helmintos, protozoários

\begin{abstract}
One hundred and fifty fecal samples from dogs with acute diarrhea (Group 1) and from 50 dogs without diarrhea (Group 2) were collected and analyzed at the Veterinary Teaching Hospital of the State University of Londrina, between October, 2004 and October 2005. From the positive samples (29.3\%) of Group 1, $72.7 \%$ were infected by only one agent, while $27.3 \%$ had mixed infection. The following genera were observed by coproparasitologic evaluation: Giardia sp. (9.3\%), Ancylostoma sp. (7.3\%), Cystoisospora (7.3\%), Entamoeba sp. (6.7\%), Toxocara sp.(4\%), Pentatrichomanas hominis (3.3\%), and Trichuris vulpis $(1.3 \%)$. From the 50 animals without diarrhea, six (12\%) were positive by coproparasitologic evaluation: two (4\%) for Ancylostoma sp., three (6\%) with Giardia sp., and one (2\%) for both Ancylostoma
\end{abstract}

\footnotetext{
1 Mestrando em Ciência Animal pelo Departamento de Medicina Veterinária Preventiva; CCA; UEL.

2 Professor titular doutor do Departamento de Medicina Veterinária Preventiva; CCA; UEL

3 Professor adjunto doutor do Departamento de Clínicas Veterinárias; CCA; UEL. e-mail: p.camargo@uel.br

* Autor para correspondência
} 
sp. and Cystoisospora sp. There was a significant relationship between infection and diarrhea $(\mathrm{P}=0,005)$, age and infection $(\mathrm{P}<0,001)$; but not between the occurrence of gastrointestinal parasites in males and females $(\mathrm{P}=0.08)$. The number of infected pure breed dogs was significantly higher than their mixed breed counterparts $(\mathrm{P}=0.01)$. The results show the importance of realizing periodic parasitological examination in dogs with or without diarrhea for the specific treatment and the implementation of prophylaxis and control methods.

Key words: Dogs, acute diarrhea, gastrointestinal parasites, helminthes, protozoa

\section{Introdução}

Os cães representam os animais de estimação que mais convivem com o homem (LEITE et al., 2004). A ligação emocional estabelecida pode trazer benefícios físicos e psicológicos, além de melhorar a integralização social de portadores de doenças imunossupressoras, idosos, crianças e pessoas com necessidades especiais (PARSLOW; JORM, 2003; McNICHOLAS et al., 2005). Porém, a proximidade com o cão de estimação resulta em maior exposição humana a agentes com potencial zoonótico (SILVA et al., 2001). Diversos parasitos gastrintestinais que utilizam o cão como hospedeiro definitivo ou intermediário, podem ser transmitidos ao homem e causar doenças (ANDRESIUK et al., 2003).

As parasitoses gastrintestinais estão entre as doenças mais freqüentes e importantes dos cães neonatos e jovens. Helmintos, como Toxocara sp. e Ancylostoma sp., devido ao seu potencial zoonótico são considerados um problema de saúde pública (SANTARÉM; GIUFFRIDA; ZANIN, 2004). A infecção no homem pelo Toxocara canis causa a síndrome de larva migrans visceral e o Ancylostoma sp. é responsável pela síndrome de larva migrans cutânea, (NUNES et al., 2000) e eventualmente por lesões viscerais (HENDRIX et al., 1996).

Protozoários, como a Giardia sp. e o Cryptosporidium sp. também são considerados agentes infecciosos importantes, tanto para cães como para pessoas. O Cryptosporidium sp., tem distribuição cosmopolita, infecta uma grande variedade de espécies animais e é relacionado a quadros de diarréia aguda em cães
(O'DONOGHUE, 1995). Por ser um agente oportunista, é uma grande preocupação relacionada a infecção em pessoas imunocomprometidas, transplantados, pacientes em quimioterapia e crianças (GUERRANT, 1997).

A ocorrência de parasitoses gastrintestinais, em cães domiciliados e errantes, tem sido estudada em diversos países (BLAGBURN et al., 1996; HACKETT; LAPPIN, 2003; TRILLOALTAMIRANO; CARRASCO; CABRERA, 2003), e em diferentes regiões do Brasil (ARAÚJO et al., 1986; COSTA et al., 1990; LEITE et al., 2004; ALVES; GOMES; SILVA, 2005). Estudos de Chieffi et al. (1976) e Guimarães Junior et al. (1996) relataram a ocorrência de parasitos gastrintestinais em amostras de fezes de cães da região de Londrina, observando infecções únicas ou associadas.

A importância clínica das parasitoses gastrintestinais em cães e o convívio próximo ao homem, principalmente quando o cão é domiciliado, gera a necessidade de se conhecer a ocorrência dos parasitos para delineamento e adoção de medidas preventivas e educativas.

Este estudo teve como objetivo verificar a ocorrência de helmintos e protozoários gastrintestinais em cães domiciliados, com diarréia, oriundos da região metropolitana de Londrina, atendidos no Hospital Veterinário Escola da Universidade Estadual de Londrina, no período de outubro de 2004 a outubro de 2005. Foi também verificada a distribuição da ocorrência de parasitismo segundo o sexo, faixa etária e definição racial dos animais. 


\section{Material e Métodos}

\section{Delineamento experimental}

Foi realizado um estudo prospectivode casos controlados de escolha aleatória baseado em população canina atendida em hospital veterinário escola, tendo comoúnico critério de seleção a presença de diarréia aguda.

\section{Animais examinados}

Foram incluídos na pesquisa 150 cães com diarréia de evolução aguda (Grupo 1), e para grupo controle, 50 cães sem diarréia (Grupo 2), todos domiciliados, escolhidos aleatoriamente entre os animais atendidos no período de outubro de 2004 a outubro de 2005 no Hospital Veterinário (HV) Escola, da Universidade Estadual de Londrina (UEL).

Dos animais do Grupo 1 (G1), 81 eram machos e 69 fêmeas, com idades variando de 30 dias a 16 anos, sendo 100 cães com raça definida e 50 sem definição racial. Dos animais do Grupo 2 (G2), 23 eram machos e 27 fêmeas, com idade variando de 45 dias a 15 anos, sendo 18 com raça definida e 32 sem definição racial.

\section{Amostragem}

Foi colhida uma amostra de fezes de cada animal e imediatamente submetida ao exame parasitológico direto (uma gota de fezes frescas, uma gota de solução salina $0,9 \%$ entre lâmina e lamínula), pela observação em microscópio óptico. O restante da amostra foi mantido a $4^{\circ} \mathrm{C}$, e no máximo em 24 horas, foram realizados os demais exames. As técnicas empregadas foram Willis e Mollay (1921), Hoffman, Pons e Janes (1934), Faust modificada (De CARLI, 2001) e Ziehl-Neelsen modificada (HENRIKSEN; POLENZ, 1981). Os exames coproparasitológicos foram realizados no Laboratório de Parasitologia Animal no Hospital Veterinário Escola da Universidade Estadual de Londrina. Os dados obtidos foram submetidos à análise estatística pelos testes do $X^{2}$ e de regressão linear (ZOLMAN, 1993).

\section{Resultados e Discussão}

Das 150 amostras dos animais do G1, 44 (29,3\%) foram positivas para uma ou mais espécies de parasitos, enquanto que das 50 do G2, seis (12\%) foram positivas (Tabela 1). A diferença de positividade entre as amostras coletadas de cães com e sem diarréia aguda foi estatisticamente significativa $(\mathrm{P}=0,005)$, demonstrando que a ocorrência de parasitismo gastrintestinal estava associada ao quadro clínico. Em contrapartida, não foi observada relação entre a proporção de infecções simples ou múltiplas $(\mathrm{P}=0,12)$.

Tabela 1. Freqüência absoluta e relativa de resultados positivos e negativos na avaliação coproparasitológica de amostras provenientes de cães domiciliados, com diarréia aguda (Grupo 1) e sem diarréia (Grupo 2), oriundos da região metropolitana de Londrina - Norte do estado do Paraná, atendidos no HV - UEL*, no período de outubro de 2004 a outubro de 2005. Londrina, 2006.

\begin{tabular}{|c|c|c|}
\hline \multirow{3}{*}{ Amostras } & \multicolumn{2}{|c|}{ Animais } \\
\hline & Grupo 1 & Grupo 2 \\
\hline & $N^{0}(\%)$ & $\mathbf{N}^{0}(\%)$ \\
\hline Negativas & $106 / 150(70,7)^{\mathrm{a}}$ & $44 / 50(88,0)^{\mathrm{a}}$ \\
\hline Positivas & $44 / 150(29,3)^{\mathrm{a}, \mathrm{c}}$ & $6 / 50(12,0)^{a, c}$ \\
\hline Infecção simples & $32 / 44(72,7)^{b}$ & $5 / 6(83,3)^{b}$ \\
\hline Infecção múltipla & $12 / 44(27,3)^{b, c}$ & $1 / 6(16,7)^{b, c}$ \\
\hline
\end{tabular}

* HV - UEL: Hospital Veterinário da Universidade Estadual de Londrina.

${ }^{a}$ Diferença estatisticamente significativa $(\mathrm{P}=0,005)$.

${ }^{\mathrm{b}}$ Diferença sem significância estatística $(\mathrm{P}=0,12)$.

c Relação estatisticamente significativa entre a ocorrência de positivos e de infecção múltipla, no G1 e no G2 (P < 0,001). 
Nas 44 amostras positivas provenientes de cães Pentatrichomonas hominis (3,3\%), Trichuris vulpis com diarréia foram encontrados Giardia sp. $(9,3 \%), \quad(1,3 \%)$, em infecções simples ou múltiplas (Tabela 2 Ancylostoma sp. (7,3\%), Cystoisospora sp. (7,3\%), e Figura 1).

Entamoeba sp. (6,7\%), Toxocara sp. (4,0\%) e

Tabela 2. Freqüência absoluta e relativa de ocorrência de parasitos intestinais em amostras de fezes de cães domiciliados, oriundos da região metropolitana de Londrina, atendidos no HV - UEL*, no período de outubro de 2004 a outubro de 2005, com diarréia aguda (Grupo 1) e sem diarréia (Grupo 2). Londrina, 2006.

\begin{tabular}{|c|c|c|}
\hline \multirow{3}{*}{ Parasitos } & \multicolumn{2}{|c|}{ Animais } \\
\hline & Grupo $1(n=150)$ & Grupo $2(n=50)$ \\
\hline & $\mathrm{N}^{0}(\%)$ & $\mathbf{N}^{0}(\%)$ \\
\hline \multicolumn{3}{|l|}{ Helmintos } \\
\hline Ancylostoma sp. & $6(4,0)$ & $2(4,0)$ \\
\hline Toxocara sp. & $2(1,3)$ & $0(0)$ \\
\hline Trichuris vulpis & $2(1,3)$ & $0(0)$ \\
\hline \multicolumn{3}{|l|}{ Protozoários } \\
\hline Giardia sp. & $10(6,7)$ & $3(6,0)$ \\
\hline Cystoisospora sp. & $6(4,0)$ & $0(0)$ \\
\hline Entamoeba $\mathrm{sp}$ & $4(2,7)$ & $0(0)$ \\
\hline Pentatrichomonas hominis & $2(1,3)$ & $0(0)$ \\
\hline Cryptosporidium sp. & $0(0)$ & $0(0)$ \\
\hline \multicolumn{3}{|l|}{ Infecções múltiplas } \\
\hline Cystoisospora sp. e Toxocara sp. & $2(1,3)$ & $0(0)$ \\
\hline Entamoeba sp. e Pentatrichomonas hominis & $2(1,3)$ & $0(0)$ \\
\hline Ancylostoma sp. e Cystoisospora sp. & $1(0,7)$ & $1(2,0)$ \\
\hline Ancylostoma sp. e Giardia sp. & $1(0,7)$ & $0(0)$ \\
\hline Ancylostoma sp. e Toxocara sp. & $1(0,7)$ & $0(0)$ \\
\hline Cystoisospora e Giardia sp. & $1(0,7)$ & $0(0)$ \\
\hline Giardia sp. e Entamoeba sp. & $1(0,7)$ & $0(0)$ \\
\hline Ancylostoma sp., Entamoeba sp. e Cystoisospora & $1(0,7)$ & $0(0)$ \\
\hline Ancylostoma sp., Entamoeba sp. e Toxocara sp. & $1(0,7)$ & $0(0)$ \\
\hline Entamoeba sp., Giardia sp. e Pentatrichomonas hominis & $1(0,7)$ & $0(0)$ \\
\hline Total & $44(29,3)$ & $6(12,0)$ \\
\hline
\end{tabular}

HV - UEL: Hospital Veterinário da Universidade Estadual de Londrina. 


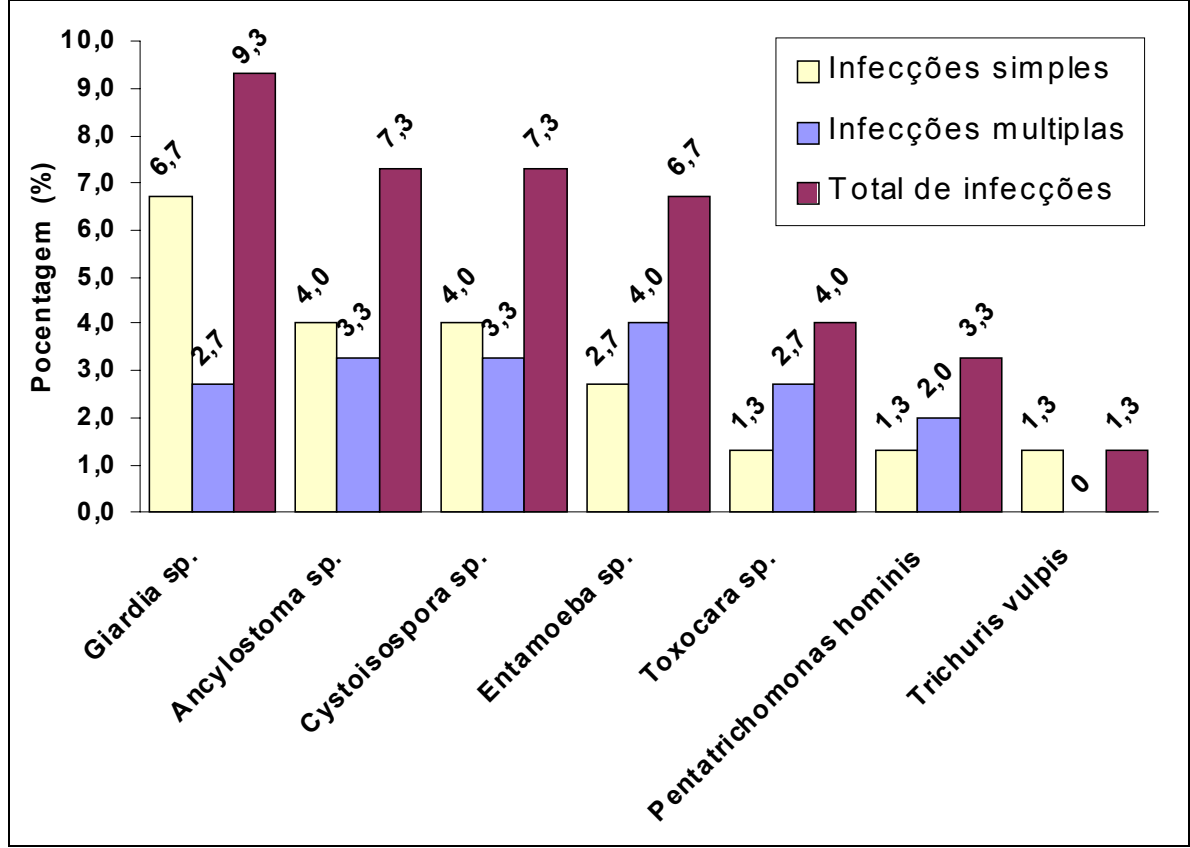

Figura 1. Percentual da ocorrência de parasitos gastrintestinais, em infecções simples e múltiplas, nas amostras de fezes de cães com diarréia (Grupo 1), provenientes da região metropolitana de Londrina, Norte do Estado do Paraná, analisadas no período de outubro de 2004 a outubro de 2005.

A ocorrência de infecções mistas é relatada com freqüência em estudos envolvendo cães com diarréia aguda (BLAGBURN et al., 1996; HACKETT; LAPPIN, 2003; VASCONCELLOS; BARROS; OLIVEIRA, 2006). A infecção por um parasito, principalmente no cão filhote ou jovem, causa debilidade orgânica que favorece a instalação de um segundo agente, porém, reflete também a infecção maternal ou contaminação ambiental por múltiplas espécies.

A Giardia sp. foi o parasito de maior ocorrência, presente em dez $(9,3 \%)$ dos cães do G1, e em três (6\%) dos cães do G2. Hackett e Lappin (2003) também relataram Giardia sp. como parasito mais encontrado em cães domiciliados do Colorado - EUA, presente em 5,6\% (4/71) dos cães com diarréia e em 5,1\% (3/59) sem diarréia. Em cães oriundos do Rio de Janeiro e Niterói, também domiciliados, a taxa de infecção por Giardia sp. foi de 1,9\% (4/212) (BRENER et al., 2005). Em contrapartida, na cidade de São Paulo foram encontradas 7,6\% (27/353) de amostras positivas também em cães domiciliados (GENNARI et al.,1999). Em Goiânia, a ocorrência do protozoário em fezes de cães domiciliados foi menor do que aquela observada em outras localidades (1,6\% ou 6/384), mesmo nos cães errantes $(0 / 50)$ (ALVES et al., 2005).

A infecção por Giardia sp. foi mais freqüente do que por helmintos como Ancylostoma sp. ou Toxocara sp., que são geralmente relatados como parasitos mais comuns em cães (GENNARI et al., 1999; BRENER et al., 2005; VASCONCELLOS; BARROS; OLIVEIRA, 2006). Todos os cães examinados no presente estudo eram domiciliados e provavelmente consomem a mesma água que seus proprietários. Considerando que a ingestão de água contaminada é a principal fonte de infecção para o homem, esses dados alertam para a necessidade de averiguar a qualidade da água consumida na região metropolitana de Londrina. O fato de ter sido encontrada também em fezes de cães sem diarréia deve ser considerado, pois mesmo aparentemente saudáveis podem, sem os devidos cuidados, representar riscos ao proprietário e sua família.

O Ancylostoma sp. foi encontrado em 11 (7,3\%) das amostras do G1 e em três (6\%) do G2. Hackett 
e Lappin (2003) em estudo similar realizado no Colorado, EUA, observaram a presença deste agente em 1,4\% (1/71) das amostras de fezes provenientes de cães com diarréia, porém não o encontraram em cães sem diarréia. No Peru, em estudo envolvendo cães domiciliados sem diarréia, Ancylostoma sp. foi encontrado em 9,2\% (15/162) das amostras (TRILLO-ALTAMIRO; CARRASCO; CABRERA, 2003). Os cães domiciliados da cidade de São Paulo (GENNARI et al., 1999) e do Rio de Janeiro (BRENER et al., 2005) apresentaram taxas de infecção maiores, 20,4\% (72/353) e 20,3\% (43/ 212) respectivamente. Vasconcellos, Barros e Oliveira (2006) identificaram Ancylostoma sp. em $34,8 \%(71 / 204)$ de amostras provenientes de cães errantes e foi o mais freqüente entre os parasitos. Alves, Gomes e Silva (2005), analisaram fezes de cães errantes e domiciliados e relataram 22\% (11/ 50) e $9,9 \%$ (38/384), respectivamente. Por estar menos expostos ao agente, os cães domiciliados apresentam menor índice de infecção.

A ocorrência do Ancylostoma observada, quando comparada a relatos anteriores sugere tendência decrescente na taxa de infecção na região de Londrina. Em 1976, Chieffi et al. (1976) estudaram a ocorrência de endoparasitos em cães domiciliados da zona urbana de Londrina e encontraram Ancylostoma sp. em 51,8\% (71/137) das amostras de fezes. Vinte anos depois, Guimarães Junior et al. (1996) relataram 39,8\% (354/889) de positividade em cães domiciliados atendidos no HV-UEL. No presente estudo foi observada ocorrência de 7,3\% $(11 / 150)$ de amostras positivas. Nas três décadas que decorreram desde a pesquisa de Chieffi et al. (1976), o vínculo afetivo entre as pessoas e seus cães vem se estreitando. Esta proximidade provavelmente resulta em dispêndio de mais atenção ao animal, que se traduz em melhor alimentação e cuidados sanitários, como administração de anti-helmintícos e programas profiláticos de controle, cuidados não dispensados aos cães errantes. A disponibilidade de fármacos de melhor qualidade, com princípio ativo eficaz, bem com a facilidade de obtenção e popularização dos tratamentos também deve ser um fator contribuinte para este decréscimo.

O protozoário Cystoisospora sp. foi encontrado em $11(7,3 \%)$ das amostras provenientes de cães com diarréia e em um (2\%) dos cães sem diarréia. No Colorado, EUA, Cystoisospora sp. estava presente em 4,2\% (3/71) de amostras oriundas de cães domiciliados com diarréia e em 1,7\% (1/59) dos sem diarréia (HACKETT; LAPPIN, 2003). Em cães domiciliados de São Paulo foi identificado em 2,5\% (9/353) das amostras (GENNARI et al.,1999), enquanto em cães errantes do Rio de Janeiro foram $5,9 \%$ (12/204) (VASCONCELLOS et al., 2006). Em Goiânia, Cystoisospora sp. foi menos encontrado em cães domiciliados $(2,6 \%$ ou $10 / 384)$ do que nos errantes $(10 \%$ ou $5 / 50)$, sendo mais freqüente em animais jovens (ALVES et al., 2005). Também no presente estudo a maior ocorrência foi observada nas fezes de animais mais jovens.

A infecção por Entamoeba sp. foi diagnosticada em $10(6,7 \%)$ dos animais com diarréia, ocorrência elevada quando comparada com estudos anteriores. Brener et al. (2005) utilizando as técnicas de Hoffman e Faust, não encontram o agente em fezes de cães colhidas no Rio de Janeiro e em Niterói. As diferenças nos resultados podem ser decorrentes de diferenças entre as técnicas laboratoriais empregadas. $\mathrm{Na}$ realização das diferentes técnicas de diagnóstico empregadas neste trabalho, o parasito foi observado somente no exame direto, porém não nos exames de Hoffman e Faust, realizados posteriormente. Isso indica que o Exame Direto de fezes frescas é o mais adequado para o diagnóstico de Entamoeba sp.

A Entamoeba sp. é considerada um agente oportunista. Neste estudo observamos que sua ocorrência foi maior $(4,0 \%)$ associada a outros agentes do que em infecções simples $(2,7 \%)$, reforçando a hipótese do oportunismo. Contudo, como Entamoeba sp. pode ser patogênica para pessoas e animais infectados podem servir de fonte de infecção para o proprietário e sua família, devese considerar a importância de sua ocorrência em cães no nosso meio. 
O Pentatrichomonas hominis foi encontrada em cinco amostras $(3,3 \%)$ de fezes dos cães com diarréia. A ocorrência deste protozoário em cães não tem sido relatada em estudos anteriores. Este parasito é comumente encontrado no exame direto de fezes logo após a coleta (SHERDING, 1996), e a falta de relatos pode ser decorrente das técnicas empregadas no diagnóstico. As amostras positivas eram provenientes de cães com idade inferior a um ano e, na maioria dos casos, o parasito estava associado a outros agentes. Esses dados estão de acordo com o descrito em Sherding (1996) e reafirmam a característica oportunista de Pentatrichomonas hominis. Além disso, deve-se considerar o potencial zoonótico do agente.

Ovos de Toxocara sp foram encontrados em seis (4\%) cães com diarréia e em nenhum dos animais sem diarréia, observação semelhante à de Hackett e Lappin (2003). A infecção foi diagnosticada apenas em cães com até um ano de idade, como o encontrado nos cães de Goiânia (ALVES; GOMES; SILVA, 2005), reforçando a importância deste parasito como agente de diarréia em filhotes e cães jovens. Maior taxa de ocorrência de Toxocara sp. em cães domiciliados foi relatada na cidade de São Paulo, observando $8,5 \%$ ou 30 em 353 animais (GENNARI et al., 1999), e no Peru, onde foi de $19,75 \%$ ou 32 em 162 cães (TRILLOALTAMIRANO; CARRASCO; CABRERA, 2003). No Rio de Janeiro, em fezes de cães errantes, o parasito também foi observado em índice maior ( $8,8 \%$ ou $18 / 204)$ do que relatado no presente trabalho (VASCONCELLOS; BARROS; OLIVEIRA, 2006). Já, em cães errantes e domiciliados da cidade de Goiânia os índices encontrados foram de 4\% (2/ 50 ) e 2,34\% (9/384), respectivamente (ALVES; GOMES; SILVA, 2005). Estas variações refletem a influência do meio de vida dos animais e da região avaliada na taxa de infecção por este agente. Assim como para outros parasitos intestinais, também demonstra o risco que cães errantes podem representar para a população humana e canina.

Semelhante a infecção por Ancylostoma sp. estudos anteriores realizados com cães da região de
Londrina mostram uma tendência decrescente. Ha 30 anos Toxocara sp. foi encontrado em 46\% (63/ 137) (CHIEFFI et al., 1976), taxa que em 1996 foi de 13,7\% (122/889) (GUIMARÃES JUNIOR et al., 1996) e neste estudo foi de apenas 4\% (6/150). O decréscimo na taxa de infecção provavelmente se deve aos mesmos motivos aventados para a infecção por Ancylostoma sp.

A menor taxa de ocorrência de infecção foi do helminto Trichuris vulpis, observado em apenas dois cães com diarréia $(1,3 \%)$, taxa semelhante à observada em cães domiciliados do Colorado, EUA (1,4\% ou 1/71) (HACHETT; LAPPIN, 2003). Taxas menores foram observadas em cães domiciliados de São Paulo (0,28\% ou 1/353) (GENNARI et al., 1999). Novamente os índices de infecção observados em cães domiciliados são menores do que os observados em cães errantes $(2,5 \%$ ou 5/204) (VASCONCELLOS; BARROS; OLIVEIRA, 2006).

A semelhança do observado para outros helmintos, a taxa de infecção por Trichuris vulpis em cães da região metropolitana de Londrina mostra uma tendência decrescente. Em 1976 ovos deste parasito foram encontrados em 13,1\% (18/137) das amostras (CHEIFFI et al., 1976); em 1996 foram 6,7\% (60/889) (GUIMARÃES JUNIOR et al., 1996) e agora apenas $1,3 \%(2 / 150)$.

O Cryptosporidium sp. tem sido considerado um agente oportunista que infecta cães com sistema imune comprometido, situação em que também pode causar doença em pessoas. As taxas de infecção em cães variam de 2,8\%, ou 10 em 353 animais (GENNARI et al., 1999) a 5,6\%, ou quatro em 71 cães (HACKETT; LAPPIN, 2003). Em Goiânia, novamente foi vista diferença importante entre cães domiciliados $(2 \%$ ou $8 / 384)$ e errantes $(6,0 \%$ ou $3 /$ 50) (ALVES; GOMES; SILVA, 2005). Em estudo anterior também envolvendo cães com diarréia atendidos no HV - UEL, Cryptosporidium sp. foi encontrado em 2,25\% (3/133) das amostras avaliadas (NAVARRO et al., 1997). Empregando técnica de diagnóstico semelhante à empregada neste estudo 
anterior não foi encontrado Cryptosporidium sp. em nenhuma das amostras. A diminuição da ocorrência deste protozoário na região de Londrina pode estar relacionada a diminuição de outros parasitos envolvidos em quadros de diarréia aguda, com os quais o Cryptosporidium sp poderia estar associado, porém, é contrário ao aumento observado na ocorrência de outros protozoários.

Nas amostras analisadas neste estudo não foi encontrado Dipylidium caninum, agente relatado em estudos anteriores feitos na região em percentuais que variaram de $2,91 \%$ a $3,82 \%$ respectivamente (CHIEFFI et al., 1976; GUIMARÃES JUNIOR et al., 1996). Esta diminuição pode estar relacionada a popularização do uso de produtos para controle de pulgas nos animais de estimação.
A ocorrência de parasitismo intestinal teve relação estatística significante $(P=0,001)$ com a faixa etária dos animais (Tabela 3). Os resultados encontrados demonstraram que o número de animais com até seis meses parasitados foi significativamente maior do que nas faixas etárias mais altas, e decrescente com o aumento da idade. Estes resultados são semelhantes a observações anteriores (OLIVEIRA et al., 1990; ALVES; GOMES; SILVA, 2005), confirmando que, por fatores relacionados à imunidade, os cães mais jovens são mais predispostos ao parasitismo (TRILLO-ALTAMIRANO; CARRASCO; CABRERA, 2003). Porém, não houve relação ( $\mathrm{P}=$ $0,62)$ da faixa etária com a ocorrência de infecção múltipla, sugerindo que a infecção por mais de um agente pode estar ligada mais a condições ambientais do que a fatores intrínsecos do animal.

Tabela 3. Ocorrência, em números absolutos e relativos, distribuídos por faixa etária, de parasitismo intestinal nos cães com diarréia (G1) atendidos no HV - UEL*, no período de outubro de 2004 a outubro de 2005. Londrina, 2006.

\begin{tabular}{|c|c|c|c|c|c|}
\hline \multirow{2}{*}{ Total de observações } & \multicolumn{5}{|c|}{ Faixa etária (meses) } \\
\hline & $0-\mid 6$ & $6--12$ & $12--24$ & $>24$ & TOTAL \\
\hline Infecções simples & $19(22,1 \%)$ & $5(16,1 \%)$ & $1(16,7 \%)$ & $7(25,9 \%)$ & $32(21,3 \%)$ \\
\hline Infecções múltiplas & $8(9,3 \%)$ & $2(6,5 \%)$ & $1(16,7 \%)$ & $1(3,7 \%)$ & $12(8,0 \%)$ \\
\hline Positivos & $27(31,4 \%)$ & $7(22,6 \%)$ & $2(33,3 \%)$ & $8(29,6 \%)$ & $44(29,3 \%)$ \\
\hline Negativos & $59(68,6 \%)$ & $24(77,4 \%)$ & $4(66,7 \%)$ & $19(70,4 \%)$ & $106(70,7 \%)$ \\
\hline Animais examinados & $86(57,3 \%)$ & $31(20,7 \%)$ & $6(4,0 \%)$ & $27(18 \%)$ & $150(100 \%)$ \\
\hline
\end{tabular}

* HV - UEL: Hospital Veterinário Escola da Universidade Estadual de Londrina.

Relacionando a distribuição dos resultados dos animais do G1 com a definição racial (Tabela 4) foi possível observar que o número de animais de raça definida parasitados foi significativamente maior $(\mathrm{P}$ $=0,01)$ do que o de animais sem raça definida. Em estudo anterior envolvendo animais da mesma região (GUIMARÃES JUNIOR et al., 1996) o número de animais sem definição racial parasitados foi maior do que de animais com raça definida. Esta discrepância pode ser apenas o resultado do aumento do número de cães com raça definida na população canina em geral, mas esta informação somente pôde ser averiguada para a nossa amostra de cães com diarréia, que não apresentou diferença significativa da população atendida no Hospital Veterinário Escola de UEL, atendida no período de outubro de 2002 a outubro de $2005(\mathrm{P}=0,305)$. Porém, a ocorrência de parasitas variou significativamente com a faixa etária, tanto nos animais sem raça definida como nos com raça definida, indicando que a positividade nas faixas etárias independe da característica racial $(\mathrm{P}<0,001)$. 
Tabela 4. Distribuição absoluta e relativa da ocorrência de parasitas nos cães (G1) atendidos no Hospital Veterinário Escola da Universidade Estadual de Londrina., no período de outubro de 2004 a outubro de 2005, com e sem raça definida. Londrina, 2006.

\begin{tabular}{cccccc}
\hline \multirow{2}{*}{ Definição racial } & \multicolumn{4}{c}{ Faixa etária (meses) } & Total \\
\cline { 2 - 5 } & $\mathbf{0}-\mid \mathbf{6}$ meses & $\mathbf{6 - | 1 2}$ meses & $\mathbf{1 2}-\mid \mathbf{2 4}$ meses & $\mathbf{2 4}$ meses & \\
\hline Com raça definida & $23 / 61(37,7 \%)^{\mathrm{a}}$ & $6 / 19(31,6 \%)^{\mathrm{b}}$ & $0 / 3(0,0 \%)^{\mathrm{c}}$ & $6 / 17(35,3 \%)^{\mathrm{d}}$ & $35 / 100(35 \%)^{\mathrm{e}}$ \\
Sem raça definida & $4 / 25(16,0 \%)^{\mathrm{a}}$ & $1 / 12(8,3 \%)^{\mathrm{b}}$ & $2 / 3(66,6 \%)^{\mathrm{c}}$ & $2 / 10(20 \%)^{\mathrm{d}}$ & $9 / 50(18 \%)^{\mathrm{e}}$ \\
\hline
\end{tabular}

a,b,c,d - estatisticamente significativo para a faixa etária mas não para a definição racial. ${ }^{a}(\mathrm{P}=0,03),{ }^{\mathrm{b}, \mathrm{c}}(\mathrm{P}<0,001),{ }^{\mathrm{d}}(\mathrm{P}=0,02)$

${ }^{\mathrm{e}}$ - cães com raça definida forram significativamente mais parasitados que os sem raça definida $(\mathrm{P}=0,01)$.

Entre os animais com diarréia, 81 eram machos e 69 fêmeas, sendo que dos machos 29 estavam parasitados $(35,8 \%)$ e das fêmeas foram $15(21,7 \%)$, diferença sem significância estatística $(\mathrm{P}=0,08)$. Esta observação é semelhante aos relatos anteriores (ALVES; GOMES; SILVA 2005; TRILLOALTAMIRANO; CARRASCO; CABRERA, 2003), confirmando que machos e fêmeas estão igualmente sujeitos ao parasitismo intestinal e a taxa de infecção está relacionada a fatores ambientais e sanitários. Convém relatar que não houve diferença significativa, para o sexo, entre a população amostral do G1 e a população atendida no Hospital Veterinário da UEL, no período de outubro de 2002 a outubro de $2005(\mathrm{P}=0,48)$.

Em resumo, neste estudo a ocorrência de parasitismo intestinal foi observada principalmente em cães com até seis meses de idade, indicando que este ainda é um problema importante para cães filhotes e jovens. Apesar da diminuição da incidência quando comparado a outros trabalhos realizados na região de Londrina, medidas de profiláticas para o controle nesta faixa, em que os cães ficam predispostos a desenvolver infecções, devem ser adotadas. Além disso, cães jovens com diarréia são potenciais disseminadores de patógenos para o ambiente e para as pessoas que convivem em maior proximidade, principalmente crianças. O estreitamento da relação do homem com seu cão de estimação resultam na melhora da qualidade de vida do animal, que recebe maior atenção quanto à alimentação, melhores cuidados sanitários e profiláticos, que são cuidados não dispensados aos cães errantes.

Na região metropolitana de Londrina tem ocorrido uma significativa diminuição da ocorrência dos parasitos mais comuns do cão, porém observou-se um aumento na diversidade de agentes, principalmente de protozoários, mesmo em cães sem diarréia. A ocorrência de protozoários representou $67,8 \%$ do total de parasitos encontrados, demonstrando sua importância como agentes de doença gastrintestinal, principalmente em cães filhotes, agentes estes que requerem medidas profiláticas, terapêuticas e ambientais específicas, além de educação da população. Além disso, a maioria dos protozoários intestinais encontrados tem potencial zoonótico. Essas constatações reafirmam a importância de realização de exames coproparasitológicos em cães com diarréia associada a tratamento específico e a medidas de controle ambiental.

O fato de Giardia sp., Ancylostoma sp. e Cystoisospora sp. terem sido encontrados em cães sem sinais de doença gastrintestinal reforça a necessidade da realização periódica de exames coproparasitológicos e tratamento específico em todos os cães, independente da presença ou não de sintomas de doença gastrentérica. 


\section{Agradecimentos}

Os autores agradecem à Profa Dra. Roberta Lemos Freire, aos técnicos Aldair Calistro de Matos e Dalva Maria Navarro Fabrício, e estagiária Roberta dos Santos Toledo pelo apoio na realização dos exames parasitológicos.

\section{Referências}

ALVES, O. F.; GOMES, A. G.; SILVA, A. C. Ocorrência de enteroparasitos em cães do município de Goiânia, Goiás: comparação de técnicas de diagnóstico. Ciência Animal Brasileira, Goiania v.6, n.2, p.127-133, 2005.

ANDRESIUK, M. V.; DENEGRI, G. M.; ESARDELLA, N.H; HOLLMANN, P. Encuesta coproparasitológico canina realizado en plazas publicas de la ciudad de Mar Del Plata, Buenos Aires, Argentina. Parasitología Latinoamericana, Santiago de Chile, v.58, n.1-2, p.17-22, 2003.

ARAÚJO, R. B.; FERREIRA, P. M.; ASSIS, C. B.; DEL CARLO, R. J.; VIEIRA, D. Helmintoses intestinais em cães da microregião de Viçosa-MG. Arquivo Brasileiro de Medicina Veterinária e Zootecnia, Belo Horizonte, v.38, n.2, p.197-203, 1986.

BLAGBURN, B. L.; LINDSAY, D. S.; VAUGHAN, J. L.; RIPPEY, N. S.; WRIGHT; J. C; LYNN, R. C.; KELCH, W. J.; RITCHIE, G. C.; HEPLER, D. I. Prevalence of canine parasites based on fecal flotation. Compendium on Continuing Education for the Practicing Veterinarian, Princeton, v.18, n.5, p.483-510, 1996.

BRENER, B.; LISBOA, L.; MATTOS, D. P. B. G.; ARASHIRO, E. K. N.; MILLAR, P. R.; SUDRÉ, A. P.; DUQUE, V. Freqüência de enteroparasitas em amostras fecais de cães e gatos dos municípios do Rio de Janeiro e Niterói. Revista Brasileira de Ciência Veterinária, Niterói, v.12, n.1/3, p.102-105, 2005.

CHIEFFI, P. P.; MÜLLER, E. E; VIOTTI, N. M. A.; MORETTI, I. G. Estudo sobre a prevalência de enteroparasitas em cães da zona urbana do município de Londrina, Estado do Paraná, Brasil. Científica, Jaboticabal, v.4, n.1, p.64-67, 1976.

COSTA, J. O.; LIMA, W. S.; GUIMARÃES, M. P.; LIMA, E. A. M. Freqüência de endo e ectoparasitos de cães capturados nas ruas de Vitória - ES - Brasil. Arquivo Brasileiro de Medicina Veterinária e Zootecnia, Belo Horizonte, v.42, n.5, p.451-452, 1990.

De CARLI, G. A. Parasitologia Clínica: seleção de métodos e técnicas de laboratório para o diagnóstico dos parasitos humanos. São Paulo: Atheneu, 2001.
GENNARI, S. M.; KASAI, N.; PENA, H. F. J.; CORTEZ, A. Ocorrência de protozoários e helmintos em amostras de fezes de cães e gatos da cidade de São Paulo. Brazilian Journal of Veterinary Research and Animal Science, São Paulo, v.36, n.2, p.87-91, 1999.

GUERRANT, R. L. Cryptosporidiosis: an emerging, highly infectious threat. Emerging Infectious diseases, Atlanta, v.3, n.1, p.51-57, 1997.

GUIMARÃES JUNIOR, J. S.; VIDOTTO, O.; YAMAMURA, M. H.; ROSS, G. M.; FONSECA, N. A. N.; PEREIRA, A. B. L. Helmintoses gastrointestinais em cães (canis familiaris) na região de Londrina-PR. Semina: Ciências Agrárias, Londrina, v.17, n.1, p.29-32, 1996.

HACKETT, T.; LAPPIN, M. R. Prevalence of enteric pathogens in dogs of north-central Colorado. Journal of the American Animal Hospital Association, Lakewood, v.39, n.1, p.52-56, 2003.

HENDRIX, C. M.; BRUCE, H. S.; KELLMAN, N. J.; HARRELSON, G.; BRUHN, B. F. Cutaneous larva migrans and enteric hookworm infections. Journal of the American Veterinary Medical Association, Schaumburg, v.209, p.1763-1767, 1996.

HENRIKSEN, S. A.; POLENZ, J. Staining of Cryptosporidia by a modified Ziehl-Neelsen technique. Acta Veterinaria Scandinavica, Copenhagen, v.22, p.294$269,1981$.

HOFFMAN, W. A.; PONS, J. A.; JANER, J. L. The sedimentation concentration method in schistostosomiase mansoni. Journal of Public Health, Local, v.9, p.238-291, 1934.

HOFFMANN, R. P.; FORTES, E.; PANDOLFO, R. A.; KAISER, J. C.; BELLÓ, A. R. R.; MOTTA NETO, A. A. Prevalência de helmintos gastrintestinais do cão errante do município de Porto Alegre, Rio Grande do Sul. Arquivos da Faculdade de Veterinária da Universidade Federal do Rio Grande do Sul, Porto Alegre, v.18, p.61-68, 1990.

LEITE, L. C.; MARINONI, L. P.; CÍRIO, S. M.; DINIZ, J. M. F.; SILVA, M. A. N.; LUZ, E.; MOLINARI, H. P.; VARGAS, C. S. G.; LEITE, S. C; ZADOROSNEI, A. C. B.; VERONESI, E. M. Endoparasitas em cães (Canis familiaris) na cidade de Curitiba-Paraná-Brasil. Archives of Veterinary Science, Curitiba, v.9, n.2, p.95-99, 2004.

McNICHOLAS, J.; GILBEY, A.; RENNIE, A.; AHMEDZAI, S.; DONO, J.; ORMEROD, E. Pet ownership and human health: a brief review of evidence and issues. British Medical Journal, v.331, p.1252-1254, 2005. Disponível em: $<$ http://bmj.bmjjournals.com/cgi/content/full/331/7527/ 1252>. Acesso em: 25 maio 2006. 
NAVARRO, I. T.; KANO, F. S.; OGAWA, L.; FREIRE, R. L.; VIDOTTO, O. Ocorrência de Cryptosporidium spp em cães com diarréia atendidos no Hospital Veterinário da Universidade Estadual de Londrina, PR, Brasil. Semina: Ciências Agrárias, Londrina, v.18, n.1, p.23-25, 1997.

NUNES, C. M.; PENA, F. C; NEGRELLI, G. B.; ANJO, C. G. S.; NAKANO, M. M.; STOBBE, N. S. Ocorrência de larva migrans na areia de áreas de lazer das escolas municipais de ensino infantil, Araçatuba, SP, Brasil. Revista de Saúde Pública, São Paulo, v.34, n.6, p.656-658, 2000.

OLIVEIRA, P. R.; SILVA, P. L.; PARREIRA, V. F.; RIBEIRO, S. C. A.; GOMES, J. B. Freqüência de endoparasitos em cães da região de Uberlândia, Minas Gerais. Brazilian Journal of Veterinary Research Animal Science, São Paulo, v.27, p.193-197, 1990.

O'DONOGHUE, P. J. Cryptosporidium and cryptosporidiosis in man and animals. International Journal of Parasitology, Oxford, v.25, n.2, p.139-195, 1995.

PARSLOW, R. A.; JORM, A. F. Pet ownership and risk factors for cardiovascular disease: another look. Medical Journal of Australia, Sydney, v.179, p.466-468, 2003.

SANTARÉM, V. A.; GIUFFRIDA, R.; ZANIN, G. A. Larva migrans cutânea: ocorrência de casos humanos e identificação de larvas de Ancylostoma spp em parque público do município de Taciba, São Paulo. Revista Brasileira de Medicina Tropical, Rio de Janeiro, v.37, n.2,p.179-181, 2004.
SHERDING, R. G. Diseases of the Colon, Retum, and Anus. In: TAMS, T. R. (Ed.) Handbook of Small Animal Gastroenterology. Phyladelfia: W.B. Saunders, 1996. p.321370.

SILVA, H. C.; CASTAGNOLLI, K. C.; SILVEIRA, D. M.; COSTA, G. H. N.; GOMES, R. A.; NASCIMENTO, A. A. Fauna helmíntica de cães e gatos provenientes de alguns municípios do Estado de São Paulo. Semina: Ciência Agrárias, Londrina, v.22, n.1, p.63-66, 2001.

TRILLO-ALTAMIRANO, M. P.; CARRASCO, A. J., CABRERA, R. Prevalência de helmintos enteroparásitos zoonóticos y factores em Canis familiaris em uma zona urbana de la ciudad de Ica, Peru. Parasitology Latinoamericana, Santiago de Chile, v.58, p,136-141, 2003

VASCONCELLOS, M. C.; BARROS, J. S. L.; OLIVEIRA, C. $\mathrm{S}$. Parasitas gastrointestinais em cães institucionalizados no Rio de Janeiro, RJ. Revista Saúde Pública, São Paulo, v.40, n.2, p.321-323, 2006.

WILLIS, H. H. A simple levitation method for the detection of hookworm ova. Medical Journal of Australia, Sydney, v.8, p.375-376, 1921.

ZOLMAN, J. F. Biostatistics: experimental design and statistical inference. New York: Oxford University Press, 1993.p.343. 
